\title{
Music Therapy: The Poor Stepchild Whose Time has Come
}

Steven A Sparr ${ }^{1^{*}}$ and Concetta M Tomaino ${ }^{2}$

${ }^{1}$ Director of Neurorehabilitation, Montefiore Medical Center, Albert Einstein College of Medicine, 111 East 210 Street, Bronx, New York, USA

${ }^{2}$ Executive Director, Institute for Music and Neurological Function, New York, USA

"Corresponding author: Steven A Sparr, Director of Neurorehabilitation, Montefiore Medical Center, Albert Einstein College of Medicine, Bronx, New York, USA, Tel: 718-920-6402; E-mail: ssparr@montefiore.org

Rec date: Mar 25, 2015; Acc date: Mar 27, 2015; Pub date: April 02, 2015

Copyright: (c) 2015 Sparr S, et al. This is an open-access article distributed under the terms of the Creative Commons Attribution License, which permits unrestricted use, distribution, and reproduction in any medium, provided the original author and source are credited.

\section{Editorial}

Music therapy provides a powerful tool for the rehabilitation of wide variety of neurological disorders including aphasia, hemiparesis, Parkinson's disease, epilepsy, visuospatial neglect and dementias of various etiologies. Yet recognition of this important modality has been very slow in coming. Music therapy remains the poor stepchild of Rehabilitation Medicine, excluded by most acute rehabilitation facilities and not reimbursed by many third party payers.

How have we arrived at this current situation?

The power of music was obvious as far back as the birth of modern Neurology when Paul Broca demonstrated that language localized to the left side of the brain. His index case, Monsieur Leborgne, had verbal expression limited to a single syllable ("tan!") and yet was able to sing la Marseillaise in its entirety! There are many examples of the ability of music to ameliorate clinical deficits that are clear from observation of neurological patients at the bedside-the patient with expressive aphasia who can sing, the frozen patient with Parkinson's disease who suddenly can walk with good cadence and arm swing when music is playing, the patient with severe dementia with little interaction with the environment who becomes animated and verbal when asked to sing or play a rhythm instrument.

Historically, however, music therapy has been slow in gaining widespread acceptance because, until recent years, the science has lagged behind the phenomenology. Localization of music in the brain proved elusive to the clinico-anatomical methods of the classical era of discovery in the late nineteenth and early twentieth centuries despite the best efforts of Neurology's Founding Fathers including Charcot, Gowers, Fischer, Feuchtwanger and others. As a result, the role of music in human cognition and disorders of musical processing have received scarce mention in medical training, including in the training of neurologists and physiatrists. If a function cannot be mapped in the brain, the attitude seemed to be, it cannot be all that important. As a result, music has been viewed as a form of "entertainment" and not as an essential cognitive function. After all, how important can a cognitive function be if patients can live completely functional lives without it?

In the past two decades a revolution has taken place as modern neuroimaging and clinical evaluation of patients with neurosurgical procedures have made significant progress in elucidating the neurobiology of music. It now clear that musical processing requires the participation of widespread cortical and subcortical networks bilaterally in the brain and has strong connectivity not only to emotional functions but to motor systems as well. It has also been demonstrated that there is a high degree of plasticity in the representation of music in the brain and that exposure to music can lead to permanent alterations in brain function [1]. In short, music sits biologically at the perfect juncture to be of use in rehabilitating the damaged brain.

In parallel with this improved understanding of the localization of musical functions in the brain have come well controlled studies of a variety of music-based therapies showing improvement of function in disorders where conventional rehabilitative modalities have had limited efficacy. One of the earliest techniques was the development of Melodic Intonation Therapy (MIT) in the 1970's [2]. MIT was shown to improve fluency of speech in patients with expressive aphasia, even long after the patients had plateaued with traditional speech therapy. Improvement was felt to be due to recruitment of right hemispheric components of language, especially prosody, which can bypass damaged left hemispheric circuits. These predicted neuroplastic changes in language production have been ultimately demonstrated with modern functional imaging in patients treated with MIT [3].

In more recent years, rhythmic stimulation has been shown to improve gait in patients with Parkinson's disease [3-5] with studies showing increased walking speed, stride length and improved stride time variability. These effects were sustained for weeks after completion of therapy. Music based therapy for patients with Parkinson's disease has demonstrated significantly greater improvements in bradykinesia, emotional function and activities of daily living compared with conventional physical therapy [6].

Altenmüller and colleagues showed improvement in speed, precision and smoothness of both fine and gross motor functions in hemiplegic stroke patients randomized to music supported therapy compared with controls treated with standard physical therapy [7]. These improvements were "hard wired" and accompanied by robust changes in event related neurophysiological parameters such as desynchronization prior to movements.

In 1993 Hughes and colleagues [8] showed reduction in epileptic form discharges in patients exposed to music; even those in coma benefited. These effects have been duplicated and both short-term and long-term reduction in seizure frequency has been demonstrated in both child and adult epileptics exposed to music [9].

Music therapy has even proven effective in the remediation of wide variety of other higher cognitive disorders many of which are not well addressed by standard physical and occupational therapies such as visuospatial neglect [10] and executive dysfunction in traumatic brain injury [11]. As a form of treatment of severe dementia [12] music therapy can lead to improvement in social interaction and level of arousal.

The results of these studies indicate that music is not a side show in human cognition, but is an intrinsic component of the human mind with powerful connections to the emotional and motor systems. Music therapy has been shown to be able to harness this powerful, plastic 
Citation: Sparr S, Tomaino MC (2015) Music Therapy: The Poor Stepchild Whose Time has Come. Int J Neurorehabilitation 2: 1000e106. doi:

Page 2 of 2

system in the remediation of a large number of neurological disorders when treatment is provided by well trained professionals capable of delivering this care [13].

After years as the poor stepchild of Rehabilitation Medicine, music therapy has come of age and should be invited to sit at the table alongside physical, occupational and speech therapies. The time has come for music therapy to be integrated into both inpatient and outpatient rehabilitation programs and to be recognized as a meaningful modality of treatment by third party payers. We now know that not only is music therapy biologically plausible, it is actually effective!

\section{References}

1. Altenmüller E, Schlaug G (2013) Neurobiological Aspects of Neurological Music Therapy. Music and Medicine 5: 210-216.

2. Albert ML, Sparks RW, Helm NA (1973) Melodic intonation therapy for aphasia. Arch Neurol 29: 130-131.

3. Schlaug G, Marchina S, Norton A (2009) Evidence for plasticity in white matter tracts of patients with chronic Broca's aphasia undergoing intense intonation based speech therapy. Ann. New York Acad. Sci. 1169:385-394.

4. Rubinstein TC, Giladi N, Hausdorff JM (2002) The power of cueing to circumvent dopamine deficits: a review of physical therapy treatment of gait disturbances in Parkinson's disease. Mov Disord 17: 1148-1160.

5. Hausdorff JM, Lowenthal J, Herman T, Gruendlinger L, Peretz C, et al. (2007) Rhythmic auditory stimulation modulates gait variability in Parkinson's disease. Eur J Neurosci 26: 2369-2375.
6. Lim I, van Wegen E, de Goede C, Deutekom M, Nieuwboer A, et al. (2005) Effects of external rhythmical cueing on gait in patients with Parkinson's disease: a systematic review. Clin Rehabil 19: 695-713.

7. Pacchetti C, Mancini, F, Aglieri, R, Fundaro, C, Martignoni E, et al., (2000) Active music therapy in Parkinson's disease: An integrative method for motor and emotional rehabilitation. Psychosom Med. 62: 386-393.

8. Altenmüller E, Marco-Pallares J, Münte,TF, Schneider S (2009) Neural Reorganization Underlies Improvement in Stroke-Induced Motor Dysfunction by Music Supported Therapy. Ann N Y Acad Sci 1169: 395-405.

9. Hughes JR, Daaboul Y, Fino JJ, Shaw GL (1998) The "Mozart effect" on epileptiform activity. Clin Electroencephalogr 29: 109-119.

10. Lin LC, Yang RC (2013) Using Music to Treat Epilepsy in Children: A Review. Music and Medicine 5: 242-247.

11. Bodak R, Malhotra P, Bernardi N, Cocchini G, Stewart S (2014) Reducing Chronic Visuo-Spatial Neglect Following Right Hemisphere Stroke Though Instrument Playing. Front. Hum. Neurosci. 8: 413-422.

12. Thaut MH, Gardiner JC, Holmberg D, Horwitz J, Kent L, et al. (2009) Neurologic Music Therapy Improves Executive Function and Emotional Adjustment in Traumatic Brain Injury Rehabilitation. Ann N Y Acad Sci. 1169: 404-416.

13. Tomaino CM (2013) Meeting the Complex needs of Persons with Dementia through Music Therapy. Music and Medicine 5: 234-241. 